\title{
Fluoroquinolone consumption and Escherichia coli resistance in Japan: an ecological study
}

\author{
Fumitaka Terahara ${ }^{1,2}$ and Hiroshi Nishiura ${ }^{1,2^{*}}$ (i)
}

\begin{abstract}
Background: The frequency of antimicrobial resistance has steadily increased worldwide, induced by inappropriate use of antibiotics in a variety of settings. We analyzed the ecological correlation between fluoroquinolone consumption and levofloxacin resistance in Escherichia coli in Japan.

Methods: We collected information on cases of E. coli resistant to levofloxacin in 2015-2016 in all 47 prefectures from the Japan Nosocomial Infections Surveillance system. Information on fluoroquinolone consumption was obtained from pharmaceutical sales data. To address potential confounding, we also collected information on the number of physicians, nurses, and medical facilities per 100,000 individuals.

Results: We identified higher fluoroquinolone consumption and higher resistance in western prefectures, and lower consumption and resistance in eastern prefectures. Multivariate analysis identified a positive correlation between fluoroquinolone consumption and levofloxacin resistance in both 2015 and 2016.

Conclusions: Fluoroquinolone consumption and levofloxacin-resistant $E$. coli are potentially associated on a nationwide scale. The relationship between the two must be elucidated using additional studies with different epidemiological designs, so that any possible counter-measures, including alternative prescription, can be considered in the future.
\end{abstract}

Keywords: Antibiotic resistance, Escherichia coli, Quinolones, Drug prescription, Epidemiology, Antimicrobial stewardship

\section{Background}

The frequency of antimicrobial resistance (AMR) has steadily increased worldwide, induced by inappropriate use of antibiotics in a variety of settings [1]. Concurrent development of new antibiotics, however, has not necessarily been accelerated, rendering the issue of resistance a global concern. The World Health Organization endorsed the Global Action Plan on Antimicrobial Resistance in May 2015, encouraging all member countries to formulate national action plans within 2 years [1]. In response, Japan issued its National Action Plan on Antimicrobial Resistance in April 2016, explicitly specifying a number of priority goals to be achieved over the next 5

\footnotetext{
* Correspondence: nishiurah@med.hokudai.ac.jp

${ }^{1}$ Graduate School of Medicine, Hokkaido University, Kita 15 Jo Nishi 7 Chome, Kita-ku, Sapporo-shi, Hokkaido 060-8638, Japan

${ }^{2}$ CREST, Japan Science and Technology Agency, Honcho 4-1-8, Kawaguchi, Saitama 332-0012, Japan
}

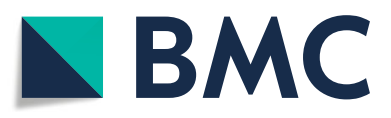

(c) The Author(s). 2019 Open Access This article is distributed under the terms of the Creative Commons Attribution 4.0 International License (http://creativecommons.org/licenses/by/4.0/), which permits unrestricted use, distribution, and reproduction in any medium, provided you give appropriate credit to the original author(s) and the source, provide a link to the Creative Commons license, and indicate if changes were made. The Creative Commons Public Domain Dedication waiver (http://creativecommons.org/publicdomain/zero/1.0/) applies to the data made available in this article, unless otherwise stated. ous surveillance initiatives and resistance countermeasures are presently underway in the country.

In its Action Plan [2], Japan aims to lower the fraction of fluoroquinolone resistance in Escherichia coli from $45 \%$ in 2014 to less than $25 \%$ by 2020 . Moreover, it aims to reduce daily fluoroquinolone use by $50 \%$ by 2020 . $E$. coli belongs to the family Enterobacteriaceae, typically found in the lower gastrointestinal tract as microbial flora, and can cause urinary tract infection and sepsis if introduced into the bloodstream. $\beta$-lactam or quinolone antibiotics are frequently the first-choice treatment for E. coli infection [3]. Quinolones are highly bioavailable, permitting oral administration and good tissue distribution, and thus may be one of the most frequently used antibiotics at both outpatient and inpatient settings. 
Increased use of antibiotics has been recognized as a major factor driving $E$. coli resistance over the last decades [4]. Fluoroquinolone resistance is easily established by point mutations in the DNA gyrase and via plasmid-mediated transfer [5], and various studies have indicated that the use of fluoroquinolone is most likely closely associated with the increase in fluoroquinolone resistance in $E$. coli [6-17]. Moreover, a few published studies reported that lowering the use of fluoroquinolone decreased the resistance proportion [18-20].

Published studies on the relationship between fluoroquinolone consumption and resistance in E. coli, however, have been mostly limited to specific healthcare facility settings, and findings on a national scale are yet to be reported. Moreover, the causal relationship must be carefully explored while adjusting for confounders. The purpose of the present study is to assess the ecological correlations between fluoroquinolone consumption and levofloxacin resistance in $E$. coli in Japan, using datasets from all 47 prefectures. Identifying the ecological association over the geographic space, one can further examine the causal link and underlying mechanisms that could potentially constitute future countermeasures.

\section{Methods}

\section{Study design}

The present study is an ecological study, exploring the relationship between fluoroquinolone consumption and resistance and exploiting geographic heterogeneities in the observed datasets in a cross-sectional manner, independently analyzing spatial datasets from 2015 and 2016. These two years were specifically examined, because only in these two years antimicrobial consumption data were accessible. For levofloxacin resistance, we collected the sensitivity testing results of levofloxacin whenever $E$. coli was detected from patients' laboratory samples. The fluoroquinolone resistance was microbiologically judged by the current breakpoint of Clinical and Laboratory Standards Institute (CLSI) to define $\mathrm{R}$ (resistant) adhering to M100-S22. The information was retrieved from the clinical laboratory section of the Japan Nosocomial Infections Surveillance (JANIS) system [21] that encompasses the microbiological testing results of all registered medical facilities. Out of the total of medical facilities with 500 beds or greater, 77.1 and $80.9 \%$ of eligible hospitals contribute to the surveillance in 2015 and 2016, respectively. Among all hospitals with 200 beds or greater, 44.5 and $48.0 \%$ of the total are registered to JANIS in 2015 and 2016. Smaller hospitals with 200 or fewer beds do not usually have an independent microbiological laboratory, and thus, only 4.4 and $6.6 \%$ of the total are registered in 2015 and
2016, respectively. All datasets originated from microbiological testing at inpatient service. The corresponding data are the sum of all relevant testing results from both outpatient and inpatient services regardless of characteristics such as infection status, colonization, or carrier.

For fluoroquinolone consumption, we used pharmaceutical sales data from wholesalers, representing the total amount of drug sold [22] by prefecture in 20152016, which has been publicly shared by the AMR Clinical Reference Centre, the National Centre for Global Health and Medicine. The sales data were standardized in accordance with the Anatomical Therapeutic Chemical classification using defined daily dose as a measurement unit, as recommended by the World Health Organization Collaborating Centre for Drug Statistics and Methodology [23]. The population-weighted consumption of oral and parenteral antimicrobials was expressed as defined daily dose per 1000 inhabitants per day (DID).

As potential confounders, we also collected the following information by prefecture for 2015-2016, and used them as explanatory variables: (i) The number of physicians per 100,000 individuals [24], (ii) the number of nurses per 100,000 individuals [25], (iii) the numbers of hospitals and clinics per 100,000 individuals [26], (iv) the number of nursing homes per 100,000 individuals [27], (v) the number of registered medical facilities in JANIS and the fraction of registered medical facilities with 500 or more inpatient beds [21], (vi) the fraction of elderly individuals in the population [28], and (vii) the average length of a hospital stay [29].

\section{Statistical analysis}

We first assessed the descriptive features of the examined variables, their distributions and summary statistics. As some variables yielded a variance-to-mean ratio greatly exceeding 1 , we consistently examined median and interquartile ranges (IQR) to summarize the distribution of all variables. For some variables with skewed distributions, we log-transformed the data to allow for an approximate normal distribution. Next, we examined univariate relationships as ecological correlations between two continuous variables, namely the fraction of levofloxacin-resistant $E$. coli cases reported and another variable. The univariate linear regression method was used to test the correlation. Finally, to adjust for confounding variables, we used multiple linear regression. Two methods were used for selecting variables to be included in the model: We included all significantly correlated variables in the multivariate model in the first method, and in the second method, a backward stepwise approach was used, applying the minimum Bayesian information criterion to select variables in the final model. 
The statistically significant level was set at $\alpha=0.05$. All statistical data were analyzed with JMP Pro version 14.0 (SAS Institute Inc., Cary, NC, USA).

\section{Results}

Table 1 shows the summary statistics of the variables examined that were considered potentially associated with the geographic heterogeneity of levofloxacin-resistant $E$. coli proportions. In 2015 and 2016, median values (and IQR) of the total isolates of $E$. coli by prefecture were 4017 (IQR: 2141-6333) and 3969 (IQR: 2744-7133), respectively. Out of those samples of $E$. coli isolates, the overall proportion of levofloxacin-resistant E. coli (hereafter, referred to as the "resistance proportion") was $37.4 \%$ in 2015 and $38.3 \%$ in 2016 . Median consumption of fluoroquinolones was 2.8 DID in both 2015 and 2016. Of the total prescriptions, the proportion of orally administered fluoroquinolones was $98.6 \%$ in 2015 and $98.5 \%$ in 2016, respectively. Figure 1 shows the geographic distributions of fluoroquinolone consumption and levofloxacin resistance. Among the 47 prefectures in Japan, the proportion of levofloxacin resistance in $E$. coli was overall lower in the eastern region than in the west, with the lowest proportions estimated in Yamagata and Aomori (27.1 and 26.2\%, in 2015 and 2016, respectively; Fig. 1a and b). The highest proportion was seen in the western Saga prefecture, and estimated at 51.1 and $55.0 \%$ in 2015 and 2016, respectively. Similarly, fluoroquinolone consumption was lower in eastern prefectures than in the west on a whole, and the lowest values were observed for Yamagata and Fukui. The highest value of fluoroquinolone consumption was observed in Tokushima, a western prefecture (Fig. 1c and d).
Table 2 summarizes the results of univariate correlation by year. The following variables were consistently and positively correlated with the proportion of levofloxacin resistance: Fluoroquinolone DID, the number of physicians, the number of nurses, and the number of hospitals and clinics. In contrast, the proportion of levofloxacin resistance was not significantly correlated with the number of nursing homes, the number of medical facilities registered in JANIS, the proportion of registered facilities with 500 or more beds, the average length of hospital stay, or the proportion of elderly individuals in the population. Figure 2 shows the crude univariate correlation between consumption of fluoroquinolone and the proportion of levofloxacin resistance. The linear regression coefficient was estimated at 0.50 and 0.52 for 2015 and 2016, respectively.

Table 3 shows the results from multivariate analyses. In advance of conducting multivariate linear regression, we identified a strong positive cross-correlation between fluoroquinolone consumption and the number of physicians (regression coefficient $=0.008$ for both 2015 and 2016 for each one-physician increase per 100,000 individuals; $p<0.001$ ). We therefore used the product of these two variables as an explanatory variable to address the possible interaction. When including all variables that were significantly correlated in the univariate analysis in the final multivariate model, no significant variables remained in the final model for either 2015 or 2016 data, and fluoroquinolone consumption was not significant $(p=0.09)$ with a large effect at 19.3 per DID increase in 2015, and not statistically significant $(p=0.16)$ with a large effect at 17.1 per DID increase in 2016. Employing the stepwise method, only fluoroquinolone consumption remained in the final model, with 5.0 and 5.5 per DID increase in 2015

Table 1 Descriptive characteristics of variables used as statistical determinants of levofloxacin resistance in Escherichia coli in Japan, 2015-2016

\begin{tabular}{|c|c|c|}
\hline \multirow[t]{2}{*}{ Variable } & \multicolumn{2}{|l|}{ Median (IQR) } \\
\hline & 2015 & 2016 \\
\hline Proportion of resistance (\%) & $37.4(33.9-42.0)$ & $38.3(35.5-42.6)$ \\
\hline Fluoroquinolone consumption (DID) & $2.8(2.4-3.2)$ & $2.8(2.4-3.3)$ \\
\hline Number of physicians per 100,000 individuals & $242.4(217.0-279.8)$ & $242.4(217.0-279.8)$ \\
\hline Number of nurses per 100,000 individuals & $965.5(817.0-1104.2)$ & $965.5(817.0-1104.2)$ \\
\hline Number of hospitals per 100,000 individuals & $7.1(5.7-10.0)$ & $7.2(5.7-10.0)$ \\
\hline Number of clinics per 100,000 individuals & $81.1(72.8-90.2)$ & $81.3(90.7-72.7)$ \\
\hline Number of nursing homes per 100,000 individuals & $7.3(6.2-8.3)$ & $6.9(5.8-8.1)$ \\
\hline Number of medical facilities included in surveillance & $21.0(15.0-37.0)$ & $26.0(17.0-40.0)$ \\
\hline Proportion of medical facilities with 500 or more beds (\%) & $20.0(15.8-27.7)$ & $17.9(12.5-25.0)$ \\
\hline Proportion of elderly in the population (\%) & $28.7(26.8-30.1)$ & $28.7(26.8-30.1)$ \\
\hline Average length of hospital stay (days) & $32.0(29.2-34.1)$ & $31.8(28.8-33.8)$ \\
\hline
\end{tabular}

DID Defined daily dose per 1000 inhabitants per day, IQR Interquartile range 

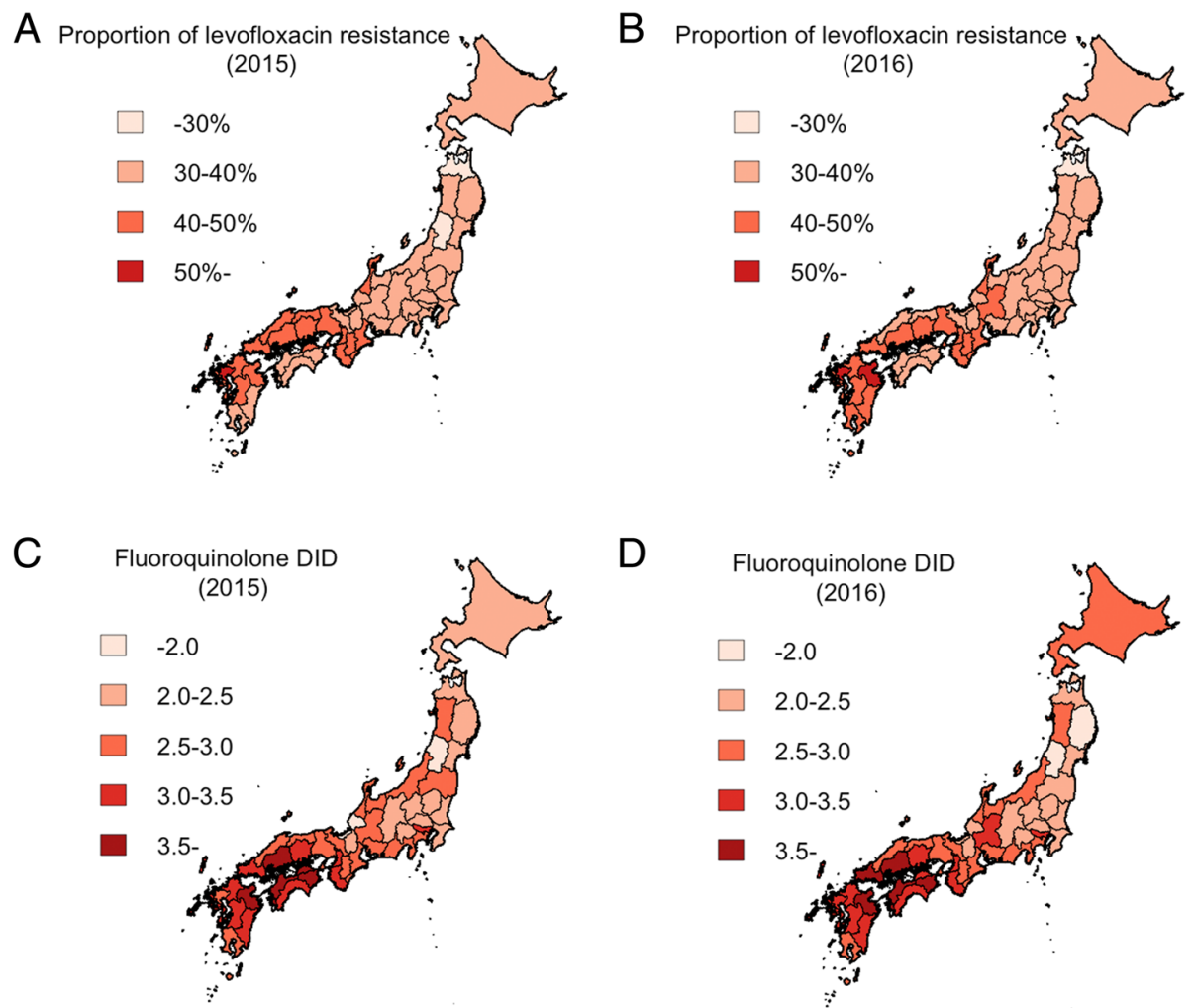

Fig. 1 Geographic distribution of fluoroquinolone consumption and levofloxacin resistance in Escherichia coli in Japan by prefecture, 2015-2016. Proportion of E. coli resistant to levofloxacin in 2015 (a) and 2016 (b). Fluoroquinolone consumption in 2015 (c) and 2016 (d), as defined daily dose per 1000 inhabitants per day (DID)

and 2016, respectively $(p<0.01$ for both years). The coefficient of determination was estimated at 0.25 for 2015 and 0.27 for 2016, with F-values of 14.8 and 17.0 , respectively ( $p<0.01$ for both years), reflecting an overall success in identifying at least some factors involved in levofloxacin resistance.

\section{Discussion}

The present study examined the ecological correlation between fluoroquinolone consumption and levofloxacinresistant $E$. coli in Japan, adjusting for potential confounding variables. Comparing fluoroquinolone consumption and levofloxacin resistance by prefecture

Table 2 Univariate correlations between the proportion of levofloxacin-resistant Esherichia coli and selected variables in Japan, 2015-2016

\begin{tabular}{|c|c|c|c|c|}
\hline \multirow[t]{2}{*}{ Variable } & \multicolumn{2}{|c|}{ Proportion resistant in 2015} & \multicolumn{2}{|c|}{ Proportion resistant in 2016} \\
\hline & Coefficient & $p$ value & Coefficient & $p$ value \\
\hline Fluoroquinolone consumption (DID) & 0.50 & $<0.01$ & 0.52 & $<0.01$ \\
\hline Number of physicians per 100,000 individuals & 0.49 & $<0.01$ & 0.42 & $<0.01$ \\
\hline Number of nurses per 100,000 individuals & 0.36 & 0.01 & 0.31 & 0.03 \\
\hline Number of hospitals per 100,000 individuals $^{\mathrm{a}}$ & 0.34 & 0.02 & 0.32 & 0.03 \\
\hline Number of clinics per 100,000 individuals & 0.43 & $<0.01$ & 0.41 & $<0.01$ \\
\hline Number of nursing homes per 100,000 individuals & -0.04 & 0.80 & -0.17 & 0.26 \\
\hline Number of medical facilities included in surveillance ${ }^{a}$ & -0.03 & 0.84 & 0.05 & 0.72 \\
\hline Proportion of medical facilities with 500 or more beds (\%) & -0.10 & 0.52 & -0.02 & 0.88 \\
\hline Average length of hospital stay (days) & 0.27 & 0.07 & 0.29 & 0.05 \\
\hline Proportion of elderly in the population (\%) & 0.04 & 0.79 & -0.04 & 0.79 \\
\hline
\end{tabular}



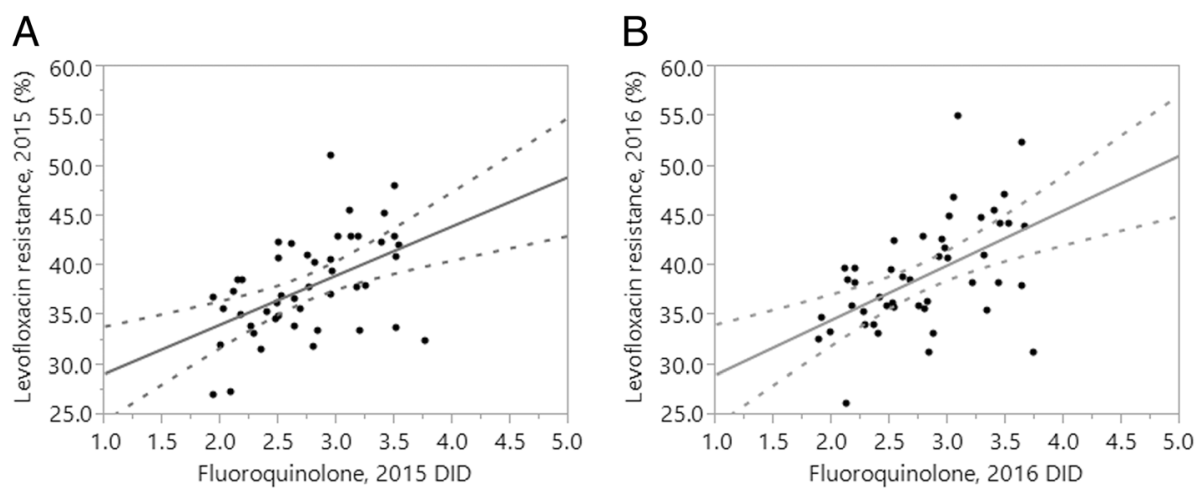

Fig. 2 Univariate correlation between fluoroquinolone consumption and levofloxacin resistance in Escherichia coli in Japan, 2015-2016. Correlation between fluoroquinolone consumption and the proportion of E. coli resistant to levofloxacin in 2015 (a) and 2016 (b), as defined daily dose per 1000 inhabitants per day (DID)

across Japan, we identified relatively higher values in the western region and lower values in the eastern region. To explain the underlying mechanisms of such geographic heterogeneities, we conducted univariate analyses, and identified not only fluoroquinolone consumption but also the numbers of physicians, nurses, hospitals, and clinics per 100,000 individuals as positively correlated with the proportion of levofloxacin-resistant $E$. coli. Multivariate analyses validated the positive correlation between fluoroquinolone consumption and levofloxacin resistance.

The present study's largest contribution is its demonstration of the link between fluoroquinolone consumption and levofloxacin resistance using nationwide surveillance data, while appropriately addressing potential confounding variables. Our study rested on a digitalized laboratory-based surveillance system, which covers $20 \%$ of healthcare facilities and $80 \%$ of medical facilities with 500 or more beds; to our knowledge, no published study has explored this relationship using a comparably large national-scale dataset. Antibiotic consumption has already been shown to be positively and strongly correlated with prescription [30], and thus, our study endorses published conclusions [6-17] that the prescription of fluoroquinolones induces the emergence (and perhaps maintenance) of resistant E. coli. Various factors may contribute to the development of levofloxacin resistance in E. coli, but our study did not identify any significant healthcare services-associated variables that were correlated with levofloxacin resistance. A high density of physicians could lead to competition and reduce hospital visits by individuals feeling unwell, in turn promoting inappropriate (and increased) prescription of fluoroquinolones. This notion was in line with the univariate analysis, but the number of physicians was removed in the final multivariate model, indicating that the information that was contained in our dataset (i.e. only the variations among the 47 prefectures) was not sufficient to establish a relationship between the number of physicians per 100,000 individuals and levofloxacin resistance. Only fluoroquinolone consumption was left in the final multivariate model using the stepwise

Table 3 Multiple regression analysis of the proportion of levofloxacin-resistant Escherichia coli in Japan, 2015-2016

\begin{tabular}{|c|c|c|c|c|c|c|c|c|}
\hline \multirow[t]{2}{*}{ Variable } & \multicolumn{4}{|c|}{ Proportion resistant in 2015} & \multicolumn{4}{|c|}{ Proportion resistant in 2016} \\
\hline & $\begin{array}{l}\text { Partial regression } \\
\text { coefficient }\end{array}$ & SE & $\mathrm{t}$ & $p$ value & $\begin{array}{l}\text { Partial regression } \\
\text { coefficient }\end{array}$ & SE & $\mathrm{t}$ & $p$ value \\
\hline Intercept constant & -23.8 & 29.8 & -0.80 & 0.43 & -14.8 & 32.9 & -0.45 & 0.66 \\
\hline Fluoroquinolone consumption (DID) & 19.3 & 11.1 & 1.75 & 0.09 & 17.1 & 12.0 & 1.43 & 0.16 \\
\hline Number of physicians per 100,000 individuals & 0.2 & 0.1 & 1.71 & 0.10 & 0.2 & 0.1 & 1.14 & 0.26 \\
\hline Number of nurses per 100,000 individuals & $-0.1<$ & $<0.1$ & -0.39 & 0.70 & $-0.1<$ & $<0.1$ & -0.37 & 0.71 \\
\hline Number of hospitals per 100,000 individuals & 1.5 & 4.2 & 0.36 & 0.72 & 2.0 & 4.8 & 0.41 & 0.69 \\
\hline Number of clinics per 100,000 individuals & $<0.1$ & 0.1 & 0.06 & 0.96 & $<0.1$ & 0.1 & 0.35 & 0.73 \\
\hline $\begin{array}{l}\text { Dummy variable (fluoroquinolone consumption } \\
\text { [DID] } \times \text { the number of physicians) }\end{array}$ & -0.1 & $<0.1$ & -1.49 & 0.14 & -0.1 & $<0.1$ & -1.08 & 0.29 \\
\hline
\end{tabular}

2015: $R^{2}=0.34, F=3.40, p<0.01$

2016: $R^{2}=0.31, F=3.00, p=0.02$

DID Defined daily dose per 1000 inhabitants per day, SE Standard error 
method, as this variable is a strong predictor of geographic heterogeneity of levofloxacin-resistant E. coli.

An important implication of the correlation between fluoroquinolone consumption and levofloxacin resistance in E. coli is that the restricted use of fluoroquinolones could potentially contribute to lowering the resistance proportion, if the resistant $E$. coli is evolutionarily unfit. The recovery of sensitivity at the population level depends on the fitness cost (i.e. transmission and self-maintenance capacity) of resistant $E$. coli. The cross-sectional design has ignored two important aspects of the emergence: (i) The induction period, i.e. the time from prescription to an increase in resistant E. coli, and (ii) the length of survival of resistant E. coli as part of the human gut microflora or elsewhere [31]. In addition to these known factors, it is worth noting that cross-resistance from the use of other antibiotics is also indicated [11, 32, 33]. The population benefit of restricted prescription requires additional epidemiological evidence and analyses. In particular, the present study was not able to account for disease burden that is associated with resistant E. coli, including urinary tract infections and respiratory tract infections, because they are not categorized as notifiable diseases in Japan. The recovery of sensitivity to fluoroquinolones should ideally be attributed to the reduction in those disease burden estimates via an appropriate epidemiological study design.

In Japan, medical expenditure has been shown to exhibit geographically heterogeneous patterns, mostly indicating higher expenditure in the west and lower in the east [34]. We have shown that fluoroquinolone consumption and resistance are no exceptions: Despite the management of healthcare services under the same system, such geographic heterogeneities are evidently observed, and no clear mechanisms were identified to explain the differences. Our study demonstrated that geographic heterogeneity of levofloxacin resistance was most likely regulated by that of fluoroquinolone consumption, and the mechanisms underlying the increase in resistance should be determined in the context of antimicrobial stewardship [35-40]. A top-down approach to restricting inappropriate prescription of antibiotics may yield evidence of the causal link suggested by our analyses.

Several limitations must be noted. First, the present study rests on an ecological study design that is known to be prone to unobserved confounding. What has been identified does not immediately lead to a causal link at the individual level. Second, the JANIS data that we mined were not accompanied by information on the type of clinical specimen or sensitivity to other antimicrobial agents. More detailed insights into levofloxacin resistance could be gained by exploring a body part from which the samples were obtained, and also examining drug sensitivity of the same bacterium to other antimicrobial agents. Third, antimicrobial consumption was measured in the present study by defined daily dose, but this approach overlooks individual heterogeneities in the prescribed dose (e.g. a lower dose in children). Published studies at the individual level can overcome this problem. Fourth, we used the number of physicians as one of variables, but the number was not stratified by hospital and primary care physicians. For the entire Japan, we have an access to the total count, i.e., there are 101,884 physicians working for clinics out of the total of 296,845 (34.3\%), but we did not have an access to the corresponding numbers by prefecture. Fifth, we used consumption and resistant frequency data, but the fluoroquinolone use at outpatient and inpatient pharmaceutical sales were not separately recorded, and moreover, microbial samples were also not stratified by inpatient and outpatient services. The distinction has an important implication for considering future restriction of antimicrobial use. Sixth, we examined only 2015 and 2016 data, because of limited access to antimicrobial consumption data. The longer the data series are collected, the more plausible that we will have a chance to elucidate the causal link, especially in the case fluoroquinolone use may be restricted from some future point in time.

In conclusion, we showed an ecological correlation between fluoroquinolone consumption and levofloxacin resistance in Japan, analyzing prefectural datasets from two recent years. The geospatial pattern of fluoroquinolone use correlated with that of resistance. The relationship between the two must be elucidated using additional studies with different epidemiological designs, so that any possible countermeasures, including alternative prescription, can be considered in the future.

\section{Conclusions}

Fluoroquinolone consumption and levofloxacin-resistant E. coli are potentially associated on a nationwide scale. The relationship between the two must be elucidated using additional studies with different epidemiological designs, so that any possible counter-measures, including alternative prescription, can be considered in the future.

\footnotetext{
Abbreviations

AMR: antimicrobial resistance; DID: defined daily dose per 1000 inhabitants per day; E. coli: Escherichia coli; IQR: Interquartile range; JANIS: Japan Nosocomial Infections Surveillance

Acknowledgements

Not applicable.

Funding

HN received funding support from the Japan Agency for Medical Research and Development (JP18fk0108050), the Japan Science and Technology
} 
Agency (JST) CREST program (JPMJCR1413), the Telecommunication Advancement Foundation, and the Japan Society for the Promotion of Science (JSPS) KAKENHI (16KT0130 and 17H04701). The funders had no role in study design, data collection and analysis, decision to publish, or preparation of the manuscript.

\section{Availability of data and materials}

Collected datasets is publicly available and can be retrieved from Japan Nosocomial Infections Surveillance (JANIS), Ministry of Health, Labour and Welfare [21] at https://janis.mhlw.go.jp/report/kensa_prefectures.html and Antimicrobial consumption surveillance, The AMR Clinical Reference Centre of National Centre for Global Health and Medicine [22] at http://amrcrc. ncgm.go.jp/surveillance/index.html.

\section{Authors' contributions}

FT conceived the study, HN conceptualized the study design, and FT collected the data. FT and HN performed statistical analyses and drafted early version of the manuscript and figures. All authors gave comments on the manuscript and approved the final version of the manuscript.

\section{Authors' information}

The authors are experts with interest in Infectious Disease Epidemiology and also in Theoretical Epidemiology, and the corresponding author acts as the chairperson and team leader of the Department of Hygiene, Hokkaido University Graduate School of Medicine.

\section{Ethics approval and consent to participate}

The present study analyzed data that is publicly available. As such, the datasets used in our study were de-identified and fully anonymized in advance, and the analysis of publicly available data without identity information does not require ethical approval [41].

\section{Consent for publication}

Not applicable.

\section{Competing interests}

The authors declare that they have no conflict of interest.

\section{Publisher's Note}

Springer Nature remains neutral with regard to jurisdictional claims in published maps and institutional affiliations.

Received: 23 August 2018 Accepted: 11 April 2019 Published online: 23 April 2019

\section{References}

1. World Health Organization (WHO). Antimicrobial resistance. Global action plan on antimicrobial resistance. Geneva: WHO; 2015. Available from: http:// apps.who.int/iris/bitstream/handle/10665/193736/9789241509763_eng.pdf

2. The Government of Japan. National action plan on antimicrobial resistance (AMR) 2016-2020. Available from: https://www.mhlw.go.jp/file/06Seisakujouhou-10900000-Kenkoukyoku/0000138942.pdf

3. Allocati N, Masulli M, Alexeyev MF, et al. Escherichia coli in Europe: an overview. Int J Environ Res Public Health. 2013;10:6235-54.

4. Bell BG, Schellevis F, Stobberingh $E$, et al. A systematic review and metaanalysis of the effects of antibiotic consumption on antibiotic resistance. BMC Infect Dis. 2014;14:13.

5. Holmes AH, Moore LS, Sundsfjord A, et al. Understanding the mechanisms and drivers of antimicrobial resistance. Lancet. 2016;387:176-87.

6. De Lastours V, Chau F, Roy C, et al. Emergence of quinolone resistance in the microbiota of hospitalized patients treated or not with a fluoroquinolone. J Antimicrob Chemother. 2014;69:3393-400.

7. Kiffer CR, Camargo EC, Shimakura SE, et al. A spatial approach for the epidemiology of antibiotic use and resistance in community-based studies: the emergence of urban clusters of Escherichia coli quinolone resistance in Sao Paulo, Brasil. Int J Health Geogr. 2011;28:10-7.

8. Cuevas O, Oteo J, Lázaro E, et al. Significant ecological impact on the progression of fluoroquinolone resistance in Escherichia coli with increased community use of moxifloxacin, levofloxacin and amoxicillin/clavulanic acid. J Antimicrob Chemother. 2011;66:664-9.
9. Hsu LY, Tan TY, Tam VH, et al. Surveillance and correlation of antibiotic prescription and resistance of gram-negative bacteria in Singaporean hospitals. Antimicrob Agents Chemother. 2010;54:1173-8.

10. Gallini A, Degris E, Desplas $M$, et al. Influence of fluoroquinolone consumption in inpatients and outpatients on ciprofloxacin-resistant Escherichia coli in a university hospital. J Antimicrob Chemother. 2010;65:2650-7.

11. Jensen US, Muller A, Brandt CT, et al. Effect of generics on price and consumption of ciprofloxacin in primary healthcare: the relationship to increasing resistance. J Antimicrob Chemother. 2010;65:1286-91.

12. Colodner R, Kometiani I, Chazan B, et al. Risk factors for community-acquired urinary tract infection due to quinolone-resistant E. coli. Infection. 2008;36:41-5.

13. Zemkova M, Kotlarova J, Merka V, et al. Emergence of fluoroquinolone resistance in Escherichia coli isolates at the department of clinical hematology. New Microbiol. 2007;30:423-30.

14. Mahamat A, Lavigne JP, Fabbro-Peray P, et al. Evolution of fluoroquinolone resistance among Escherichia coli urinary tract isolates from a French university hospital: application of the dynamic regression model. Clin Microbiol Infect. 2005;11:301-6.

15. Kern W, Steib-Bauert M, de With K, et al. Fluoroquinolone consumption and resistance in haematology-oncology patients: ecological analysis in two university hospitals 1999-2002. J Antimicrob Chemother. 2005;55:57-60.

16. Urbánek K, Kolár M, Strojil J, et al. Utilization of fluoroquinolones and Escherichia coli resistance in urinary tract infection: inpatients and outpatients. Pharmacoepidemiol Drug Saf. 2005;14:741-5.

17. Hsueh PR, Chen WH, Luh KT, et al. Relationships between antimicrobial use and antimicrobial resistance in gram-negative bacteria causing nosocomial infections from 1991-2003 at a university hospital in Taiwan. Int J Antimicrob Agents. 2005;26:463-72.

18. Cai T, Verze P, Brugnolli A, et al. Adherence to European Association of Urology guidelines on prophylactic antibiotics: an important step in antimicrobial stewardship. Eur Urol. 2016;69:276-83.

19. Wong PH, von Krosigk M, Roscoe DL, et al. Antimicrobial co-resistance patterns of gram-negative bacilli isolated from bloodstream infections: a longitudinal epidemiological study from 2002-2011. BMC Infect Dis. 2014; $14: 393$.

20. Gottesman BS, Carmeli Y, Shitrit $P$, et al. Impact of quinolone restriction on resistance patterns of Escherichia coli isolated from urine by culture in a community setting. Clin Infect Dis. 2009;49:869-75.

21. Japanese Ministry of Health, Labour and Welfare. Japan Nosocomial Infections Surveillance (JANIS). Annual open report by prefecture 2015 and 2016. Available from: https://janis.mhlw.go.jp/report/kensa_prefectures.html

22. The AMR Clinical Reference Centre of National Centre for Global Health and Medicine. Antimicrobial consumption surveillance. Available from: http:// amrcrc.ncgm.go.jp/surveillance/index.html

23. World Health Organization (WHO) Collaborating Centre for Drug Statistics and Methodology. ATC/DDD Index 2018. Available from: https://www. whocc.no/atc_ddd_index/

24. Japanese Ministry of Health, Labour and Welfare. Survey of Physicians, Dentists and Pharmacists 2016. Available from: https://www.mhlw.go.jp/ english/database/db-hss/dl/spdp_2016.pdf (details in Japanese).

25. Japanese Ministry of Health, Labour and Welfare. Report on Public Health Administration and Services 2015 and 2016. Available from: https://www. mhlw.go.jp/english/database/db-hss/dl/rophas_2016_year.pdf (details in Japanese).

26. Japanese Ministry of Health, Labour and Welfare. Summary of static/ dynamic survey of medical institutions and hospital report 2014. Dynamic survey in 2015 and 2016. Available from: https://www.mhlw.go.jp/english/ database/db-hss/mih_report_2014.html (details in Japanese).

27. Japanese Ministry of Health, Labour and Welfare. Results of "survey of institutions and establishments for long-term care" for 2016. The number of nursing homes per 100,000 people in 2015 and 2016. Available from: https://www.mhlw.go.jp/english/database/db-hss/dl/siel-2016-02.pdf (details in Japanese).

28. Japanese Cabinet Office. Annual Report on the Aging Society: 2017. 2015 aging rate (\%). Available from: http://www8.cao.go.jp/kourei/english/ annualreport/2017/pdf/c1-1.pdf

29. Japanese Ministry of Health, Labour and Welfare. Results of "survey of institutions and establishments for long-term care" for 2016. Average lengths of hospitalization days in 2015 and 2016. Available from: https:// www.mhlw.go.jp/english/database/db-hss/dl/siel-2016-02.pdf (details in Japanese). 
30. Yamasaki $\mathrm{D}$, Tanabe $\mathrm{M}$, Muraki $\mathrm{Y}$, et al. The first report of Japanese antimicrobial use measured by National Database Based on health insurance claims data (2011-2013): comparison with sales data, and trend analysis stratified by antimicrobial category and age group. Infection. 2018; 46:207-14.

31. De Lastours V, Bleibtreu A, Chau F, et al. Quinolone-resistant Escherichia coli from the faecal microbiota of healthy volunteers after ciprofloxacin exposure are highly adapted to a commensal lifestyle. J Antimicrob Chemother. 2013;69:761-8.

32. Tsukamoto N, Ohkoshi Y, Okubo T, et al. High prevalence of cross-resistance to aminoglycosides in fluoroquinolone-resistant Escherichia coli clinical isolates. Chemotherapy. 2013;59:379-84.

33. Han JH, Nachamkin I, Tolomeo P, et al. Risk factors for efflux pump overexpression in fluoroquinolone-resistant Escherichia coli. J Infect Dis. 2012;206:1597-603.

34. Japanese Ministry of Health, Labour and Welfare. Healthcare insurance database. Analysis of different regions. Available from: https://www.mhlw. go.jp/file/06-Seisakujouhou-12400000-Hokenkyoku/0000095909.pdf (in Japanese).

35. Davey P, Marwick CA, Scott CL, et al. Interventions to improve antibiotic prescribing practices for hospital inpatients. Cochrane Database Syst Rev. 2017:CD003543. https://doi.org/10.1002/14651858.CD003543.pub4.

36. Honda H, Ohmagari N, Tokuda Y, et al. Antimicrobial stewardship in inpatient settings in the Asia Pacific region: a systematic review and metaanalysis. Clin Infect Dis. 2017;64:S119-26.

37. Schuts EC, Hulscher ME, Mouton JW, et al. Current evidence on hospital antimicrobial stewardship objectives: a systematic review and meta-analysis. Lancet Infect Dis. 2016;16:847-56.

38. Drekonja DM, Filice GA, Greer N, et al. Antimicrobial stewardship in outpatient settings: a systematic review. Infect Control Hosp Epidemiol. 2015;36:142-52

39. Dellit TH, Owens RC, McGowan JE Jr, et al. Infectious Diseases Society of America and the Society for Healthcare Epidemiology of America guidelines for developing an institutional program to enhance antimicrobial stewardship. Clin Infect Dis. 2007:44:159-77.

40. Barlam TF, Cosgrove SE, Abbo LM, et al. Implementing an antibiotic stewardship program: guidelines by the infectious diseases society of America and the society for healthcare epidemiology of America. Clin Infect Dis. 2016;62:e51-77.

41. Ministry of Health, Labour and Welfare, Japan. Ethics rules for clinical studies. Tokyo: Ministry of Health, Labour and Welfare, 2009. Available from: https:/ www.mhlw.go.jp/general/seido/kousei/i-kenkyu/rinsyo/dl/gigisyoukai.pdf

Ready to submit your research? Choose BMC and benefit from:

- fast, convenient online submission

- thorough peer review by experienced researchers in your field

- rapid publication on acceptance

- support for research data, including large and complex data types

- gold Open Access which fosters wider collaboration and increased citations

- maximum visibility for your research: over $100 \mathrm{M}$ website views per year

At BMC, research is always in progress.

Learn more biomedcentral.com/submissions 